\title{
Recurrencia clínica en pacientes con puentes secuenciales en cirugía de revascularización coronaria
}

\author{
Clinical recurrence in patients with sequential bridges in coronary artery bypass grafting \\ Mario Gómez-Sánchez¹, Roberto Perezgrovas-Olaria²*, Gabriel García-Garnica², Eduardo Bucio-Reta ${ }^{1}$ y \\ Mario E. Rendón-Macías ${ }^{2}$
}

${ }^{1}$ Departamento de Investigación de Terapia Intensiva y Cirugía Cardiotorácica, Instituto Nacional de Cardiología Ignacio Chávez; ${ }^{2}$ Escuela de Medicina Universidad Panamericana. Ciudad de México, México

\section{Resumen}

Objetivo: Comparar las tasas de recurrencia de revascularización coronaria (cirugía o intervención coronaria percutánea), nuevo evento isquémico o muerte en pacientes con puentes secuenciales y con puentes simples. Método: Cohortes ambispectivas de pacientes sometidos a cirugía de revascularización coronaria secuencial $(n=111)$ o simple $(n=145)$ entre el 1 de enero de 2013 y el 31 de diciembre de 2017. Pacientes mayores de 18 años en un primer procedimiento de revascularización, con circulación extracorpórea. Para el seguimiento se realizó revisión del expediente o comunicación telefónica hasta el 9 de febrero de 2019. Se investigaron los siguientes desenlaces: reintervención por isquemia coronaria, nuevo evento isquémico documentado o muerte atribuida a cardiopatía isquémica; también se obtuvieron curvas de sobrevida. Resultados: La proporción de recurrencia según la técnica quirúrgica no fue estadísticamente diferente: secuencial $6.5 \%$ (intervalo de confianza del 95\% [IC95\%]: 2.6-12.6\%) contra simple 4.8\% (IC95\%: 2-9.7\%; $p=0.60$; análisis bayesiano $\mathrm{BF}_{10}=0.37$; evidencia moderada a no diferencia), todos por nuevo evento isquémico y un fallecimiento por grupo. No hubo diferencias en el tiempo de seguimiento: secuencial 59 meses (IC95\%: 56-62) y simple 66 meses (IC95\%: 64-68). No encontramos diferencia en las tasas de incidencia de recurrencia: secuencial 1.99 eventos $/ 10^{3}$ meses-paciente contra simple 1.47 (hazard ratio: 1.34; IC95\%: 0.47-3.8; $p=0.58$ ). El tiempo promedio de pinzamiento y de circulación extracorpórea por puente fue menor en el grupo de puentes combinados (41.44 minutos de circulación extracorpórea y 24.69 minutos de pinzamiento/puente) que en el de puentes simples (43 minutos en circulación extracorpórea y 26.4 minutos de pinzamiento/ puente) cuando se ajusta al promedio de puentes colocados (simples 2.7 y secuencial 3.25; $p<0.001$ ); sin embargo, no se encontró significancia estadística ( $p=0.7$ ). Conclusión: Ambos procedimientos tuvieron una baja incidencia de recurrencia de eventos clínicos, sin diferencias entre las técnicas quirúrgicas.

Palabras clave: Puentes secuenciales. Recurrencia clínica. Revascularización coronaria.

\begin{abstract}
Objective: To compare the recurrence rates of revascularization (redo CABG or PCI), new ischemic event or death in patients with simple grafts and patients with sequential grafts. Method: Study design is an ambispective cohort of patients that underwent CABG by sequential grafting $(n=111)$ or simple grafting $(n=145)$ between January $1^{\text {st }}, 2013$ and December $31^{\text {st }}$, 2017. Patients had to be 18 years old at the time of surgery, undergoing their first on-pump CABG. The clinical record of

Correspondencia:

*Roberto Perezgrovas-Olaria

E-mail: beto.pzgvs@gmail.com

Fecha de recepción: 26-02-2020

Fecha de aceptación: 19-03-2020 DOI: 10.24875/ACM.20000099

Disponible en internet: 23-12-2020 Arch Cardiol Mex. 2020;90(4):467-474 www.archivoscardiologia.com

1405-9940 / @ 2020 Instituto Nacional de Cardiología Ignacio Chávez. Publicado por Permanyer. Este es un artículo open access bajo la licencia CC BY-NC-ND (http://creativecommons.org/licenses/by-nc-nd/4.0/).
\end{abstract}


every patient was carefully reviewed and patients who had incomplete follow-up in external consultation were contacted by telephone in order to obtain data about ischemia related reintervention (CABG or PCl), new documented ischemic event or death caused by coronary artery disease, Kaplan-Meier estimators were calculated. Results: The proportion of recurrence depending on technique was not statistically different: sequential (6.5\% [CI95\% 2.6-12.6\%] versus simple 4.8\% [CI95\% $2-9.7 \%], p=0.60$, Bayesian analysis $B F_{10}=0.37$; moderate evidence to no difference), each one related to new ischemic event and one death per group. There were no differences in follow-up time (sequential 59m [Cl95\% 56-62] simple 66m [CI95\% 64-68]). No difference was found regarding recurrence incidence rates; sequential 1.99 events $\times 10^{3}$ months-patient, versus simple 1.47 (HR=1.34; Cl95\% 0.47-3.8, $p=0.58$ ). Pump and cross-clamping times were lower for sequential technique (41.44 min; 24.69 min respectively) versus simple technique (43 min; 26.4 min respectively) with a $p=0.7$ after adjusting to mean grafts per surgery (2.7 simple; 3.25 sequential, $p<0.001)$. Conclusions: Both techniques had a low incidence of clinical recurrence, without significant differences between procedures.

Key words: Sequential grafts. Clinical recurrence. Coronary artery bypass graft surgery.

\section{Introducción}

La cirugía de revascularización coronaria (CRC) ha representado el tratamiento ideal de la cardiopatía isquémica en pacientes con enfermedad trivascular o afección proximal de la arteria coronaria izquierda y la arteria descendente anterior, por la marcada disminución de la mortalidad y del riesgo de reintervenciones a largo plazo en los pacientes candidatos a la cirugía con respecto al intervencionismo coronario percutáneo ${ }^{1-4}$. Por otra parte, la CRC ofrece otros beneficios adicionales al intervencionismo coronario percutáneo, como son la corrección de la angina de pecho en un porcentaje superior al $90 \%$ de los pacientes sometidos al procedimiento ${ }^{5}$, así como una mayor tasa de permeabilidad del injerto a medio y largo plazo, lo cual se relaciona con una menor necesidad de repetir el proceso de revascularización y, con ello, se evitan los riesgos inherentes a este ${ }^{6}$.

Aun cuando es clara la ventaja de la CRC sobre el intervencionismo coronario percutáneo, existen controversias respecto a qué vasos deben utilizarse para revascularizar el sistema de irrigación coronario. La guía de 2011 de la American College of Cardiology Foundation/American Heart Association (ACCF/AHA) para la cirugía de revascularización coronaria sugieren como recomendación de clase I que, en aquellos casos en que sea posible, se utilice la arteria mamaria interna izquierda como hemoducto para revascularizar la arteria descendente anterior, con un nivel de evidencia $B^{7}$. Por otra parte, el resto de las recomendaciones de esta guía con respecto a la elección del segundo hemoducto en una CRC completa (todos los vasos epicárdicos $\geq 1.5 \mathrm{~mm}$ con una disminución $\geq 50 \%$ del diámetro en al menos una vista de angiografía ${ }^{8}$ ) son de clase II o de clase III en el caso del uso de puentes arteriales para revascularizar la arteria coronaria derecha si esta tiene una estenosis $<90 \%{ }^{7}$.
Para pacientes menores de 70 años, la estrategia óptima de revascularización es usar la arteria radial o la arteria mamaria interna derecha como segundo hemoducto, ya que en este grupo de pacientes la CRC con múltiples hemoductos arteriales es la principal meta. En los pacientes con edad $\geq 70$ años, la arteria radial y la arteria mamaria interna derecha deben ser usadas de manera selectiva ${ }^{9}$.

El uso de la vena safena se ha reservado principalmente para realizar puentes en la arteria coronaria derecha cuando la estenosis es $<90 \%$, y se contraindica el uso de hemoductos arteriales. Uno de los motivos por los que no se prefiere el uso de la vena safena es porque el porcentaje de estos injertos que se encuentran permeables al año de la cirugía es del 75$90 \%$, y del $50-60 \%$ a los 10 años 7,10 , aun cuando se realice manejo farmacológico intensivo con hipolipidemiantes y antiagregantes plaquetarios ${ }^{4}$. Por otra parte, más del $90 \%$ de los puentes arteriales se encuentran permeables a los 10 años de la intervención ${ }^{4,7,10}$.

Los mecanismos más importantes por los que se produce el fallo del injerto de la vena safena son principalmente tres, y cada uno de ellos predomina en distintos periodos de tiempo: la trombosis aguda es la principal causa de la falla del injerto en los primeros 30 días después de la cirugía, la neohiperplasia de la íntima predomina entre el primer mes y los 2 años de la CRC, y finalmente, la ateroesclerosis es la principal causa de la falla de los puentes después de los 2 años $^{11}$. El fallo de los injertos venosos se produce por una disrupción de la irrigación de la vena que lleva a hipoxia y genera acumulación de radicales libres que favorecen el deterioro del injerto ${ }^{4}$. Además, los efectos mecánicos por el cambio de presión y el trauma quirúrgico aceleran la disfunción del injerto ${ }^{11}$.

En determinados casos, la obtención de injertos arteriales puede derivar en un ligero incremento del riesgo 
de desarrollar complicaciones locales o sistémicas. Un ejemplo de lo antes mencionado es la mayor incidencia de infecciones mediastinales por el uso de ambas arterias mamarias internas en algunos pacientes con factores de riesgo descritos en la literatura (diabetes mellitus, obesidad y enfermedad pulmonar obstructiva crónica), dado que cuando se utilizan estos hemoductos como injertos pediculados hay una disminución de la irrigación de la pared esternal ${ }^{4,6,8,12}$. Otro ejemplo se observa en el uso de la arteria radial como injerto, debido a que puede cursar con parestesias en el brazo del que se obtiene la arteria en aproximadamente un tercio de los pacientes, aunque estos síntomas no cobran tanta importancia debido a que generalmente son transitorios y autolimitados cuando la colateralidad es adecuada ${ }^{13}$, por lo que debe realizarse la prueba de Allen y acompañarla de una segunda prueba, como ultrasonido Doppler, oximetría de pulso o toma de la presión digital con obstrucción de la arteria radial antes de utilizarla como injerto en un procedimiento de revascularización ${ }^{6}$.

El uso de la arteria mamaria interna izquierda para irrigar la arteria descendente anterior se fundamenta en dos aspectos principalmente: 1) su distribución anatómica privilegiada, que permite alcanzar la arteria descendente anterior en la mayoría de los pacientes sin tener que colocar puentes directamente desde la aorta ${ }^{5}$, lo cual disminuye el riesgo de embolia por placas de ateroesclerosis adheridas a la pared de la aorta ascendente ${ }^{8,14}$; y 2) el escaso desarrollo de ateroesclerosis a largo plazo, ya que se ha documentado que menos del $4 \%$ de las arterias mamarias internas desarrollan ateroesclerosis y que solo el $1 \%$ genera ateroesclerosis de significancia hemodinámica?.

Los mecanismos intrínsecos por los que la arteria mamaria interna no desarrolla ateroesclerosis son bien conocidos y han sido ampliamente estudiados, encontrando que factores estructurales, como una menor cantidad de fenestraciones en el endotelio y una menor permeabilidad de las uniones intercelulares, aunado a una serie de factores bioquímicos, como una mayor síntesis de moléculas antitrombóticas (sulfato de heparina y activador tisular del plasminógeno) y una producción incrementada de óxido nítrico, son aspectos fundamentales en la resistencia de este vaso a la ateroesclerosis ${ }^{4}$. Otro punto importante es que, al tener un diámetro similar al de las arterias coronarias, el flujo en los sitios de anastomosis no se vuelve turbulento, con lo que se evita la agregación plaquetaria y la formación de trombos ${ }^{4}$.

Finalmente, se ha descrito que la colocación de injertos arteriales (en particular de arteria mamaria interna y de arteria radial) tiene un efecto protector sobre las arterias coronarias nativas, ya que se ha observado una disminución en la progresión de la ateroesclerosis coronaria en comparación con los puentes realizados con vena safena ${ }^{10}$.

Todo lo anterior señala que es mejor realizar una revascularización coronaria más completa empleando tantos injertos arteriales como sea posible, con el fin de mantener su permeabilidad a largo plazo y detener el desarrollo de placas de ateroma en las arterias coronarias ${ }^{10}$. Emplear una técnica de colocación de puentes secuenciales, la cual consta de dos o más anastomosis distales realizadas con un mismo hemoducto, podría facilitar el desarrollo de revascularizaciones más completas en un menor tiempo quirúrgico cuando se compara con los puentes simples, en los que solo se realiza una anastomosis distal por cada hemoducto empleado ${ }^{15,16}$, lo cual disminuiría los tiempos de pinzamiento aórtico y de circulación extracorpórea, que son predictores importantes de morbilidad y mortalidad en cirugía cardiaca ${ }^{17,18}$.

En un metaanálisis llevado a cabo por Li, et al. ${ }^{19}$ se encontró que el riesgo absoluto de oclusión de los injertos venosos secuenciales era del $13.56 \%$, comparado con un riesgo de oclusión del $19.18 \%$ en el grupo de puentes simples.

Uno de los principales motivos por los que los puentes secuenciales han sido criticados es porque se piensa que la oclusión proximal del puente principal supone un mayor riesgo para el paciente, debido a que, en caso de comprometerse el flujo sanguíneo durante un infarto, la cantidad de miocardio afectada sería mayor que si solo se obstruyera el flujo de un injerto, como ocurriría con un puente simple ${ }^{19}$.

Este estudio pretende ser de utilidad para la toma de decisiones terapéuticas en cuanto a qué tipo de injerto favorece una revascularización completa del sistema de irrigación coronario de los pacientes, tomando como referencia la recurrencia de eventos clínicos con el paso del tiempo. La hipótesis sustentada en este trabajo es que la recurrencia de eventos clínicos con los puentes secuenciales en cirugía de revascularización coronaria es menor que con los puentes simples. El objetivo general de este estudio es determinar si los adultos con cardiopatía isquémica sometidos a cirugía de revascularización coronaria mediante puentes secuenciales tienen una menor recurrencia de eventos clínicos que los revascularizados utilizando puentes simples. 


\section{Método}

Se revisaron los expedientes de 378 pacientes sometidos a cirugía de revascularización coronaria entre el 1 de enero de 2013 y el 31 de diciembre de 2017. Se excluyeron inicialmente los pacientes que hubieran fallecido en el posoperatorio inmediato o tardío por causas distintas a una falla de los injertos colocados. Los pacientes incluidos en el estudio se dividieron en dos grandes grupos para determinar la recurrencia de eventos clínicos: 1) los que tuvieran un seguimiento completo en consulta externa y 2) los que hubieran interrumpido su seguimiento en consulta externa. Al primer grupo se le dio seguimiento a través de las notas clínicas de la consulta externa, mientras que al segundo grupo se le aplicó un cuestionario diseñado específicamente para evaluar la recurrencia de eventos clínicos por vía telefónica, eliminando todos aquellos pacientes a quienes no se les pudiera aplicar el cuestionario. Finalmente, se reportaron los eventos de acuerdo con el tipo de puente colocado en cirugía, y se establecieron dos grupos finales: puentes únicamente simples $(n=145)$ y puentes secuenciales o combinados (al menos uno simple y uno secuencial; $\mathrm{n}=111)$ (Tablas 1 y 2).

En todo momento se mantuvo la total confidencialidad de los datos de los pacientes y nunca se divulgó información de ninguno de ellos (Fig. 1).

\section{Resultados}

El análisis de los datos demográficos mostró que ambos grupos eran sumamente homogéneos al comparar los porcentajes de pacientes que tenían un factor de riesgo asociado para desarrollar enfermedad coronaria (Tabla 1). La principal diferencia encontrada se relaciona con los tiempos quirúrgicos: en el grupo de puentes simples, el tiempo medio de pinzamiento aórtico fue de $71.37 \pm 23$ minutos, mientras que en el grupo de combinado/secuencial fue de $80.3 \pm 24 \mathrm{mi}-$ nutos $(p=0.0035)$. De igual manera, se encontró que el tiempo promedio de circulación extracorpórea en el grupo simple fue de $116.24 \pm 30$ minutos, contrastando con el tiempo del grupo combinado, calculado en $134.78 \pm 34$ minutos $(p<0.001)$. Por otra parte, se encontró que el número promedio de puentes colocados en el grupo de puentes simples fue de 2.7, frente a 3.25 en el grupo combinado ( $p<0.001)$. Finalmente, al analizar de forma individualizada lo anterior, encontramos que el tiempo promedio por cada puente tiende a ser menor en el grupo de puentes combinados (41.44

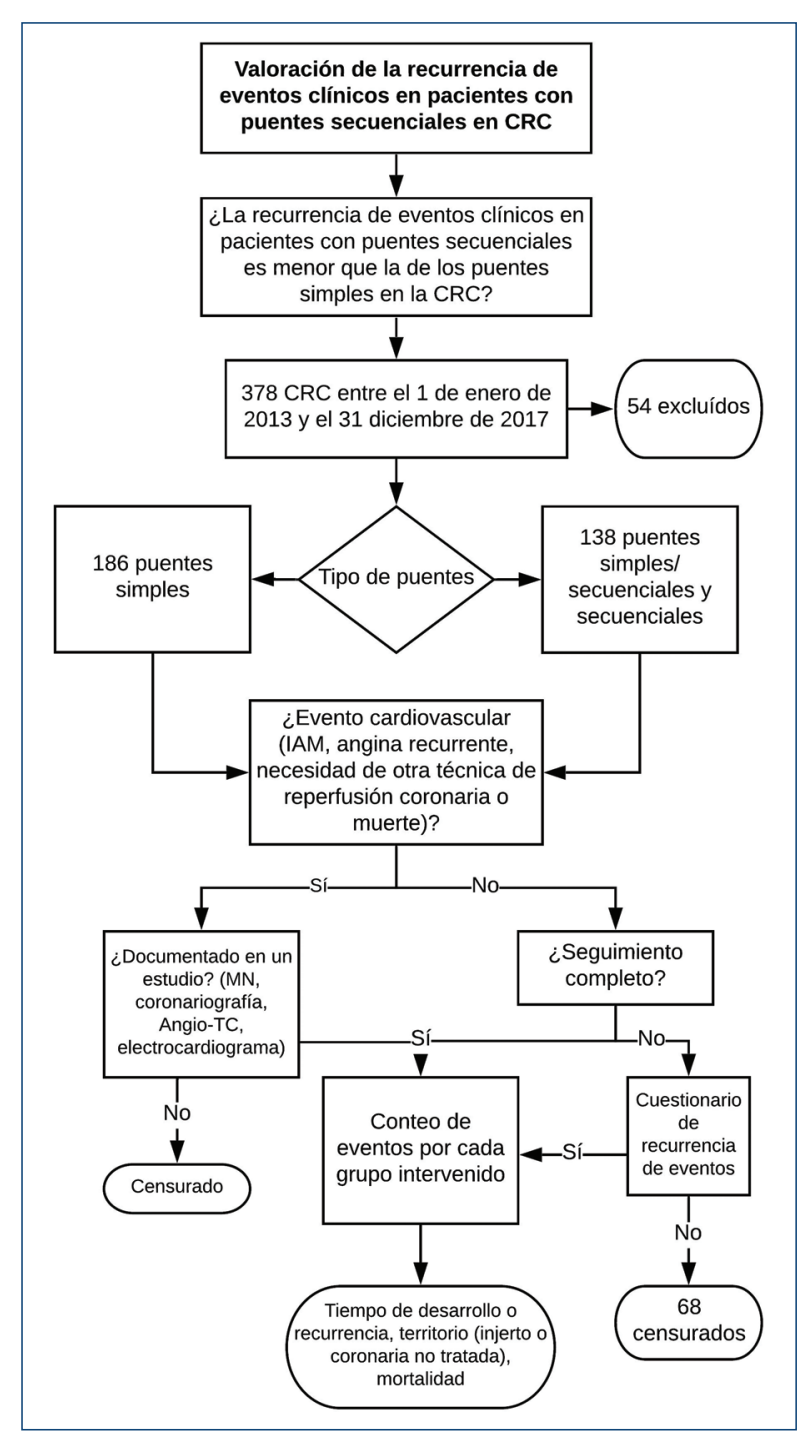

Figura 1. Diagrama de flujo que ilustra la metodología empleada. Angio-TC: angiografía por tomografía computarizada; CRC: cirugía de revascularización coronaria; IAM: infarto agudo de miocardio.

minutos en circulación extracorpórea y 24.69 minutos de pinzamiento por cada puente), comparado con el grupo de puentes simples (43 minutos en circulación extracorpórea y 26.4 minutos de pinzamiento por cada puente); sin embargo, no se encontró significancia estadística entre ambos grupos ( $p=0.7$ ) (Figs. 2 y 3). La proporción de recurrencia (eventos clínicos, infarto o mortalidad) según la técnica quirúrgica no fue estadísticamente diferente: secuencial 6.5\% (intervalo de confianza del 95\% [IC95\%]: 2.6-12.6) y simple 4.8\% (IC95\%: 2-9.7\%; $p=0.60$; análisis bayesiano $\mathrm{BF}_{10}=0.37$; evidencia moderada a no diferencia); todos por nuevo evento isquémico y un fallecimiento por 
Tabla 1. Características demográficas de los pacientes según el tipo de puente

\begin{tabular}{|c|c|c|}
\hline \multirow[t]{2}{*}{ Variables } & Puentes simples y secuenciales $(\mathrm{n}=111$ ) & Puentes simples ( $n=145$ ) \\
\hline & n $(\%)$ & n (\%) \\
\hline Media de edad (años) & 60.9 & 62.0 \\
\hline Obesidad & $23(20.7)$ & $38(26.2)$ \\
\hline Hipertensión & $65(58.6)$ & $103(71)$ \\
\hline Tabaquismo & $62(55.9)$ & $75(51.7)$ \\
\hline Diabetes mellitus & 55 (49.5) & 78 (53.8) \\
\hline Dislipidemia & $53(47.8)$ & $62(42.8)$ \\
\hline $\begin{array}{l}\text { Enfermedad renal crónica: } \\
\text { Estadio } 3 \\
\text { Estadio } 4 \\
\text { Estadio } 5\end{array}$ & $\begin{array}{c}6(5.4) \\
0 \\
0\end{array}$ & $\begin{array}{c}2(1.4) \\
0 \\
1(0.7)\end{array}$ \\
\hline $\begin{array}{l}\text { Clase funcional (NYHA): } \\
\text { I } \\
\text { II } \\
\text { III } \\
\text { IV }\end{array}$ & $\begin{array}{c}52(46.9) \\
49(44.1) \\
9(8.1) \\
1(0.9)\end{array}$ & $\begin{array}{c}60(41.4) \\
75(51.7) \\
8(5.5) \\
2(1.4)\end{array}$ \\
\hline $\begin{array}{l}\text { Clase insuficiencia cardiaca congestiva: } \\
\text { B } \\
\text { C } \\
\text { Enfermedad arterial periférica } \\
\text { Infarto previo } \\
\text { Angioplastia previa } \\
\text { Enfermedad trivascular } \\
\text { FEVI media (\%) } \\
\text { Media EuroSCORE }\end{array}$ & $\begin{array}{c}1(0.9) \\
1(0.9) \\
0 \\
63(56.8) \\
18(16.2) \\
86(77.4) \\
52.92 \\
2.44\end{array}$ & $\begin{array}{c}3(2.1) \\
0 \\
1(0.7) \\
74(51) \\
27(18.6) \\
123(84.8) \\
52.9 \\
2.08\end{array}$ \\
\hline $\begin{array}{l}\text { EuroSCORE: } \\
\text { Bajo }(0-2 \%) \\
\text { Intermedio }(2-5 \%) \\
\text { Alto }(>5 \%)\end{array}$ & $\begin{array}{l}74(66.7) \\
23(20.8) \\
14(12.7)\end{array}$ & $\begin{array}{c}96(66.2) \\
40(27.6) \\
9(6.2)\end{array}$ \\
\hline $\begin{array}{l}\text { Tipo de cirugía: } \\
\text { Electiva } \\
\text { Urgencia }\end{array}$ & $\begin{array}{c}103(92.7) \\
8(7.2)\end{array}$ & $\begin{array}{c}140(96.6) \\
5(3.4)\end{array}$ \\
\hline $\begin{array}{l}\text { Tipo de cardiopatía isquémica: } \\
\text { Angina estable } \\
\text { Angina inestable } \\
\text { SCASEST } \\
\text { SCACEST } \\
\text { Arritmia } \\
\text { Miocardiopatía dilatada } \\
\text { Media de sangrado (ml) }\end{array}$ & $\begin{array}{c}43(38.8) \\
18(16.3) \\
34(30.6) \\
15(13.5) \\
1(0.9) \\
0 \\
609.48\end{array}$ & $\begin{array}{c}45(31) \\
34(23.5) \\
44(30.3) \\
21(14.5) \\
0 \\
1(0.69) \\
630.77\end{array}$ \\
\hline
\end{tabular}

FEVI: fracción de eyección del ventrículo izquierdo; NYHA: New York Heart Association; SCACEST: Síndrome coronario agudo con elevación del segmento ST; SCASEST: Síndrome coronario agudo sin elevación del segmento ST.

grupo (Fig. 4). No hubo diferencias en el tiempo de seguimiento: secuencial 59 minutos (IC95\%: 56-62) y simple 66 minutos (IC95\%: 64-68). No encontramos diferencia en las tasas de incidencia de recurrencia: secuencial 1.99 eventos $/ 10^{3}$ meses-paciente y simple 1.47 eventos $/ 10^{3}$ meses-paciente (hazard ratio: 1.34 ; IC95\%: 0.47-3.8; $p=0.58$ ).

\section{Discusión}

En un estudio realizado por Mehta, et al. ${ }^{15}$, el uso de puentes secuenciales empleando la vena safena como hemoducto en CRC se asoció con una mayor falla del injerto a 1 año (obstrucción $>75 \%$ de la luz arterial) y con una mayor incidencia de eventos 
Tabla 2. Cuestionario para la evaluación de la recurrencia de eventos clínicos asociados a enfermedad coronaria Cuestionario para evaluación de recurrencia de eventos clínicos en pacientes revascularizados

\begin{tabular}{l|l} 
Nombre: & Número de expediente: \\
\hline
\end{tabular}

Angina de pecho

1. ¿Ha experimentado después de la cirugía un dolor de pecho opresivo similar a antes de la operación? (Frío, postprandio o esfuerzo físico posibles detonantes)

Tiempo: No tengo angina (CCS 0)

Angina solo con actividad extenuante o prolongada (CCS 1)

Angina con caminata acelerada sin pendiente. En pendientes o subir más de un bloque de escaleras (CCS 2)

Angina al caminar a ritmo normal 1-2 cuadras 0 un bloque de escaleras (CCS 3 )

Angina incluso con esfuerzo ligero o en reposo (CCS 4)

\section{Disnea}

2. Sensación de falta de aire al caminar:

Tiempo: No me falta el aire con la actividad habitual (NYHA I)

Me falta el aire con la actividad habitual (NYHA II)

Me falta el aire al caminar distancias cortas, una cuadra o 20-100 metros (NYHA III)

Me falta el aire en reposo (NYHA IV)

Toma de medicamentos

3. ¿Está tomando sus medicamentos?

3.1 Antiagregantes plaquetarios (aspirina, aspirina + clopidogrel o clopidogrel)

3.2 Estatinas

3.3 Betabloqueadores

\section{Desarrollo de ICC}

4. ¿Ha sentido que al dormir le falta el aire súbitamente, lo que le hace despertar?

5. ¿Es necesario que usted duerma con más de una almohada para que no sienta que se ahoga?

\section{Estado de ánimo}

6. ¿Cómo le hace sentir anímicamente la salud de su corazón desde que fue operado?

Bien, mi estado de ánimo ha mejorado desde el procedimiento

No ha habido cambios en mi estado de ánimo

Mal, mi estado de ánimo ha empeorado desde el procedimiento

ICC: Insuficiencia cardiaca congestiva

clínicos (infarto agudo de miocardio, necesidad de otra revascularización coronaria o muerte) a 5 años desde el procedimiento, en comparación con el uso de puentes simples del mismo hemoducto, lo cual no se vio reflejado en nuestro estudio, ya que la mayor parte de los hemoductos obstruidos en nuestra experiencia fueron arteriales y no venosos, simples y no secuenciales (Fig. 5). En contraste con lo anterior, en el estudio publicado por Kim et al..$^{20}$ se encuentra que la permeabilidad de los puentes secuenciales a mediano plazo es superior a la de los puentes simples, lo cual tampoco se vio de una manera clara en nuestro estudio, ya que los resultados extrapolados al tipo de puente individualizado fueron muy similares y no significativos. Por otra parte, Ouzounian et al. ${ }^{16}$ reportaron que el uso de puentes secuenciales no es un factor 


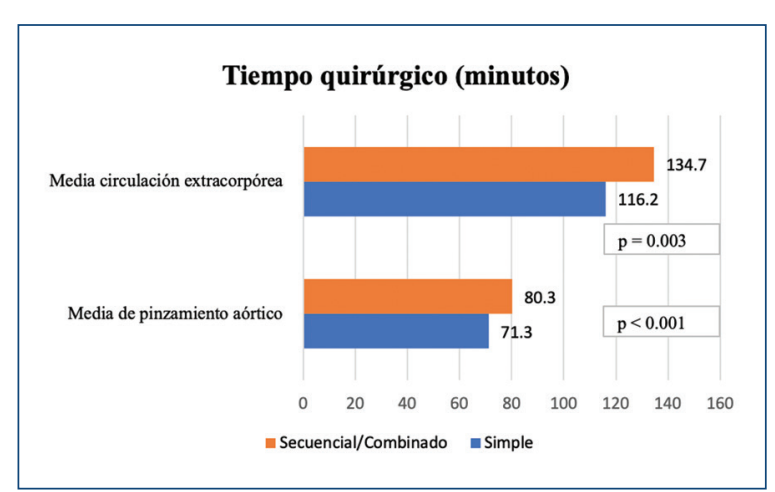

Figura 2. Comparación de los tiempos de circulación extracorpórea y de pinzamiento sin ajuste a injertos.

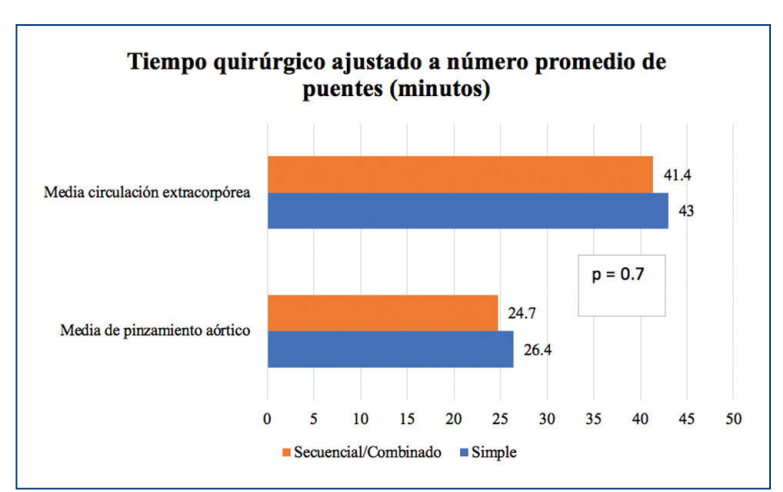

Figura 3. Comparación de los tiempos de circulación extracorpórea y de pinzamiento con ajuste a injertos promedio por grupo.

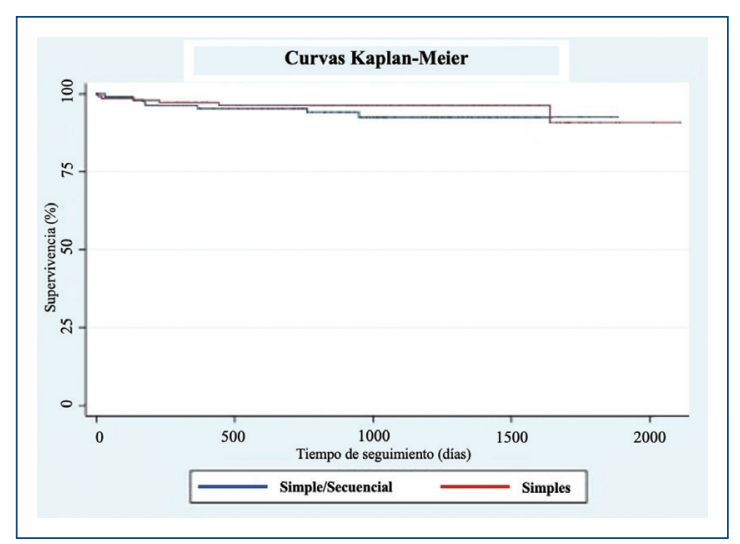

Figura 5. Estimadores de sobrevida en ambos grupos.

\section{Conclusiones}

El uso de puentes simples o de puentes secuenciales en la CRC no presenta diferencias significativas en cuanto a la recurrencia de eventos clínicos en los pacientes. Consideramos que la toma de decisiones terapéuticas con respecto a la técnica de revascularización debe ser individualizada en cada paciente. De igual manera, se demostró que los puentes secuenciales permiten realizar una revascularización más completa, y que esto podría ser de gran utilidad en aquellos pacientes que tengan un número limitado de puentes con las características adecuadas para la colocación de un injerto que se mantenga permeable a lo largo del tiempo.

\section{Financiamiento}

La presente investigación no ha recibido ninguna beca específica de agencias de los sectores público, comercial o sin ánimo de lucro. puede saberse el grado de obstrucción real de las coronarias. 


\section{Conflicto de intereses}

Los autores declaran que no existen conflictos de intereses.

\section{Responsabilidades éticas}

Protección de personas y animales. Los autores declaran que para esta investigación no se han realizado experimentos en seres humanos ni en animales.

Confidencialidad de los datos. Los autores declaran que han seguido los protocolos de su centro de trabajo sobre la publicación de datos de pacientes.

Derecho a la privacidad y consentimiento informado. Los autores declaran que en este artículo no aparecen datos de pacientes.

\section{Bibliografía}

1. Habib R, Dimitrova K, Badour S, Yammine M, El-Hage-Sleiman A Hoffman D. CABG versus PCI. JACC. 2015;66:1417-27.

2. Weintraub W, Grau-Sepulveda M, Weiss J, O'Brien S, Peterson E, Kolm $P$, et al. Comparative effectiveness of revascularization strategies. N Engl J Med. 2012;366:1467-76.

3. Sipahi I, Akay H, Dagdelen S, Blitz A, Alhan C. Coronary artery bypass grafting vs percutaneous coronary intervention in multivessel disease - reply. JAMA Int Med. 2014;174:1007.

4. Otsuka F, Yahagi K, Sakakura K, Virmani R. Why is the mammary artery so special and what protects it from atherosclerosis? Ann Cardiothorac Surg. 2013;2:519-26.

5. Pevni D, Mohr R, Lev-Ran O, Paz Y, Kramer A, Frolkis I, et al. Technical aspects of composite arterial grafting with double skeletonized internal thoracic arteries. Chest. 2003;123:1348-54.

6. Aldea G, Bakaeen F, Pal J, Fremes S, Head S, Sabik J, et al. The Society of Thoracic Surgeons clinical practice guidelines on arterial conduits for coronary artery bypass grafting. Ann Thorac Surg. 2016;101:801-9.
7. Hillis L, Smith P, Anderson J, Bittl J, Bridges C, Byrne J, et al. 2011 ACCF/ AHA guideline for coronary artery bypass graft surgery: a report of the American College of Cardiology Foundation/American Heart Association Task Force on Practice Guidelines. Circulation. 2011:124:e652-e735.

8. Kolh P, Windecker S, Alfonso F, Collet J, Cremer J, Falk V, et al. 2014 ESC/EACTS guidelines on myocardial revascularization. Eur J Cardiothorac Surg. 2014;46:517-92.

9. Tranbaugh R, Schwann T, Swistel D, Dimitrova K, Al-Shaar L, Hoffman D, et al. Coronary artery bypass graft surgery using the radial artery, right internal thoracic artery, or saphenous vein as the second conduit. Ann Thorac Surg. 2017;104:553-9.

10. Dimitrova K, Hoffman D, Geller C, Dincheva G, Ko W, Tranbaugh R. Arterial grafts protect the native coronary vessels from atherosclerotic disease progression. Ann Thorac Surg. 2012;94:475-81.

11. Harskamp R, Lopes R, Baisden C, de Winter R, Alexander J. Saphenous vein graft failure after coronary artery bypass surgery. Ann Surg. 2013;257:824-33.

12. Dorman M, Kurlansky P, Traad E, Galbut D, Zucker M, Ebra G. Bilateral internal mammary artery grafting enhances survival in diabetic patients: a 30 -year follow-up of propensity score-matched cohorts. Circulation. 2012;126:2935-42.

13. Holman W, Davies J, Lin J, Wang Y, Goldman S, Bakaeen F, et al. Consequences of radial artery harvest. JAMA Surg. 2013;148:1020-3.

14. Moss E, Puskas J, Thourani V, Kilgo P, Chen E, Leshnower B, et al. Avoiding aortic clamping during coronary artery bypass grafting reduces postoperative stroke. J Thorac Cardiovascr Surg. 2015;149:175-80.

15. Mehta R, Ferguson T, Lopes R, Hafley G, Mack M, Kouchoukos N, et al. Saphenous vein grafts with multiple versus single distal targets in patients undergoing coronary artery bypass surgery: one-year graft failure and five-year outcomes from the Project of Ex-Vivo Vein Graft Engineering via Transfection (PREVENT) IV Trial. Circulation. 2011;124:280-8.

16. Ouzounian M, Hassan A, Yip AM, Buth KJ, Baskett RJ, Ali IS, et al. The impact of sequential grafting on clinical outcomes following coronary artery bypass grafting. Eur J Cardiothorac Surg. 2010;38:579-84.

17. Al-Sarraf N, Thalib L, Hughes A, Houlihan M, Tolan M, Young V, et al. Cross-clamp time is an independent predictor of mortality and morbidity in low- and high-risk cardiac patients. Int J Surg. 2011;9:104-9.

18. Salis S, Mazzanti V, Merli G, Salvi L, Tedesco C, Veglia F, et al. Cardiopulmonary bypass duration is an independent predictor of morbidity and mortality after cardiac surgery. J Cardiothorac Vasc Anesth. 2008;22:814-22.

19. Li J, Liu Y, Zheng J, Bai T, Liu Y, Wang X, et al. The patency of sequential and individual vein coronary bypass grafts: a systematic review. Ann Thorac Surg. 2011;92:1292-8.

20. Kim H, Lee T, Kim J, Cho W, Jung S, Chung C, et al. The impact of sequential versus single anastomoses on flow characteristics and midterm patency of saphenous vein grafts in coronary bypass grafting. J Thorac Cardiovasc Surg. 2011;141:750-4 